\title{
Antimicrobial photodynamic therapy combined with periodontal treatment for metabolic control in patients with type 2 diabetes mellitus: study protocol for a randomized controlled trial
}

Erika Elisabeth Evangelista ${ }^{1}$, Cristiane Miranda França $a^{1,2,4^{*}}$, Priscila Veni ${ }^{1,3}$, Tamires de Oliveira Silva ${ }^{1}$, Rafael Moredo Gonçalves ${ }^{1}$, Verônica Franco de Carvalho ${ }^{3}$, Alessandro Melo Deana ${ }^{1}$, Kristianne P S Fernandes ${ }^{1,3,4}$, Raquel A Mesquita-Ferrari ${ }^{1,2,4}$, Cleber P Camacho ${ }^{2,5}$, Sandra Kalil Bussadori ${ }^{1,3,4}$, Letícia Heineck Alvarenga ${ }^{1}$ and Renato Araujo Prates ${ }^{1,3^{*}}$

\begin{abstract}
Background: The relationship between diabetes mellitus (DM) and periodontal disease is bidirectional. DM is a predisposing and modifying factor of periodontitis, which, in turn, worsens glycemic control and increases proteins found in the acute phase of inflammation, such as C-reactive protein. The gold standard for the treatment of periodontal disease is oral hygiene orientation, scaling and planing. Moreover, systemic antibiotic therapy may be employed in some cases. In an effort to minimize the prescription of antibiotics, photodynamic therapy (PDT) has been studied as an antimicrobial technique and has demonstrated promising results. The aim of the proposed study is to determine whether PDT as a complement to periodontal therapy (PT) is helpful in the metabolic control of individuals with type 2 diabetes and the reduction of acute-phase inflammatory markers.

Methods/Design: The patients will be randomized using a proper software program into two groups: 1) PT + placebo PDT or 2) PT + active PDT. All patients will first be examined by a specialist, followed by PT performed by two other healthcare professionals. At the end of each session, PDT (active or placebo) will be administered by a fourth healthcare professional. The following will be the PDT parameters: diode laser ( $660 \mathrm{~nm})$; power output = $110 \mathrm{~mW}$; exposure time $=90 \mathrm{~s}$ per point $\left(9 \mathrm{~J} /\right.$ point); and energy density $=22 \mathrm{~J} / \mathrm{cm}^{2}$. The photosensitizer will be methylene blue $(50 \mu \mathrm{g} / \mathrm{mL})$. The patients will be re-evaluated 15, 30, 90 and 180 days after treatment. Serological examinations with complete blood count, fasting glucose, glycated hemoglobin and salivary examinations to screen for tumor necrosis factor alpha, interleukin 1, interleukin 6, ostelocalcin, and osteoprotegerin/RANKL will be performed at each evaluation. The data will be statistically evaluated using the most appropriate tests.
\end{abstract}

Discussion: The results of this study will determine the efficacy of photodynamic therapy as an adjuvant to periodontal treatment in diabetic patients.

Trial registration: The protocol for this trial was registered with Clinical Trials registration number NCT01964833 on 14 October 2013.

Keywords: Antimicrobial, Clinical trial, Diabetes mellitus, Periodontitis, Methylene blue, Photosensitizer, Photodynamic inactivation, Red laser

\footnotetext{
* Correspondence: cristianepatologia@uninove.br; pratesra@uninove.br

${ }^{1}$ Program in Biophotonics Applied to Health Sciences, University Nove de Julho, UNINOVE, Rua Vergueiro 235/249 - Liberdade, São Paulo, SP 01504-001, Brazil

Full list of author information is available at the end of the article
} 


\section{Background}

The term 'diabetes mellitus' (DM) describes a metabolic disorder with a multifactor etiology characterized by chronic hyperglycemia, with alterations in the metabolism of carbohydrates, lipids and proteins due to deficient insulin secretion and/or insulin action [1]. DM is a major public health problem worldwide, with a prevalence rate of 246 million individuals, which is expected to reach 366 million by the year 2030 [1]. Moreover, the World Health Organization (WHO) projects a twofold increase in deaths related to diabetes between 2005 and 2030. In Brazil, approximately 10 million individuals have diabetes [2]. The aim of treatment for type 2 diabetes mellitus (T2DM) is to balance glycemic, metabolic and cardiovascular control to prevent the development of diabetes symptoms and avoid complications. This requires a coordinated multidisciplinary approach centered on the patient [1].

Periodontal disease is considered the sixth most common complication in individuals with diabetes [1]. Periodontitis is an infectious disease characterized by the destruction of the dental support structures, which leads to attachment loss, bone resorption and consequent tooth loss [3-6]. The relationship between DM and periodontal disease is bidirectional. Severe periodontitis generally coexists with diabetes and inadequate glycemic control can contribute to as much as a 2.8-fold greater risk of developing periodontal disease. Moreover, periodontal therapy (PT) reduces both systemic inflammation and resistance to insulin [7-9].

Periodontal disease is an oral infection caused by microorganisms and has been under extensive investigation regarding its microbial content. In fact, it is caused by a complex bacterial biofilm attached to the tooth and the ability of the periodontopathogens to colonize the subgingival sites is an important virulence factor. The main objective of PT is to control bacterial infection and, consequently, it is to help bone loss progression and excessive inflammatory response as well. Thus, PT is focused on scaling and root planing associated with patient oral hygiene homecare [10].

The destruction of the periodontium is triggered in response to the microbial challenge to the biofilm (dental plaque) accumulated on the tooth surface. More than $10^{8}$ bacteria and 150 different species can be found in $1 \mathrm{~mm}^{3}$ of biofilm weighing $1 \mathrm{mg}$. Eleven of these species are associated with periodontitis $[11,12]$. Dental plaque, especially Porphyromonas gingivalis, activates the immune system through Toll-like receptors, which are responsible for the recognition of pathogens and the regulation of both inflammation and bone metabolism. These receptors and cytokines related to bone resorption (interleukin (IL)-1, IL-6, and osteoprotegerin (OPG)/receptor activator of nuclear factor kappa-B ligand
(RANKL)) are altered in individuals with diabetes and have a direct association with bone loss [13].

Randomized clinical trials have shown that nonsurgical PT (oral hygiene counseling, scaling, polishing and root planing) leads to a significant reduction in plasma levels of acute-phase proteins, such as C-reactive protein and fibrinogen $[14,15]$. The best results in studies that associate systemic markers of diabetes and inflammation with PT are found when some type of antimicrobial therapy, especially doxycycline, is employed. However, the American Academy of Periodontology (AAP) recommends caution in the use of antibiotics for PT due to the side effects, the risk of favoring resistant microorganisms and difficulty in completely reaching the dental biofilm, which further favors the selection of microorganisms [5]. Antimicrobial therapy is a field of investigation that faces constant challenges due mainly to the increase in risk factors related to infection. The development of resistance to antimicrobial agents in pathogenic microorganisms is an evolutional process initiated by exposure to these drugs, which makes this issue all the more challenging. The indiscriminant use of antibiotics in recent decades has accelerated the natural selection of resistant microbial strains by allowing the rapid evolution of the genetic recombination of resistance and mutation factors [16-18]. In Brazil, the National Health Surveillance Agency (ANVISA) has adopted a number of measures aimed at monitoring microbial resistance in healthcare services and preventing the situation from becoming worse. Such actions have the direct participation of the Pan American Health Organization (PAHO), which held a conference with a number of countries in 2005 to discuss and define strategies aimed at combating microbial resistance [18].

Besides efforts at minimizing the consequences of this phenomenon, it is essential to find alternative forms of treatment that are both effective and accessible. In this context, photodynamic therapy (PDT) has emerged as a possibility for the treatment of different diseases of significant clinical importance [19-23]. This therapeutic resource is employed to cause cell death based on the use of substances with photosensitizing properties and the administration of light at an appropriate wavelength. The photodynamic process leads to the formation of free radicals and singlet oxygen, which have cytotoxic effects that can lead to the death of microorganisms [24]. PDT has demonstrated antimicrobial action in organized biofilms, especially against Porphyromonas gingivalis $[22,25,26]$.

The objectives of this work are: a) to assess the efficacy of PDT as an antimicrobial complement to PT in individuals with T2DM; b) investigate whether PT associated with PDT affects glycemic control; c) investigate whether PDT in combination with PT has a local effect 
on inflammatory marker levels and osteocalcin, OPG/ RANKL in the crevicular fluid.

\section{Methods/Design}

A randomized, parallel, double-blind, clinical trial will be conducted involving 26 patients recruited from the periodontal clinics of the Nove de Julho University (UNINOVE) (Brazil), who will be randomly allocated to two groups. The project for the study was approved by the Human Research Ethics Committee of the university (Number 375,454) on 14 August 2014 and it is identified in ClinicalTrials.gov as NCT01964833. Patients who meet the eligibility criteria and who agree to participate will sign a statement of informed consent approved by the Research Ethics Committee of the university.

\section{Inclusion criteria}

The inclusion criteria comprise T2DM patients aged 18 years or older with adequate glycemic control $[1,2,18]$; chronic periodontitis [5]; presence of at least 15 teeth and at least four teeth with bleeding upon probing and a probing depth greater than $4 \mathrm{~mm}[27,28]$; and under follow-up with an endocrinologist.

\section{Exclusion criteria}

The exclusion criteria comprise current smokers or regular smoking 12 months prior to enrollment; patients with anemia; active cancer; history of antibiotic therapy in the previous six months; history of antiinflammatory therapy in the previous three months; clotting disorder (use of anticoagulant, presence of liver disease, thrombocytopenia and immunosuppression); those currently pregnant; and those currently undergoing orthodontic treatment.

\section{Experimental groups and study design}

The sample size was calculated based on previous studies in the literature using data related to differences in mean and standard deviation values [29], determining a minimum of 13 patients with T2DM required to detect significant differences in clinical variables between evaluation times. Thirteen patients will be randomly allocated to each experimental group: Group 1 - PT (oral hygiene counseling, root scraping and planing and tooth polishing) [5] + placebo PDT; Group 2 - PT + active PDT. Evaluations will be performed at baseline as well as 15, 30, 90 and 180 days after the completion of treatment. Fig. 1 displays the flowchart of the study.

\section{Randomization of patients and allocation concealment}

Randomization of the patients to the different groups will be performed with the appropriate software program. Only the lead researcher will have access to the randomization list. Allocation to the two groups will be carried out in blocks of five at a proportion of 1:1. Each patient will receive a sealed envelope to deliver to the operator in charge of the PDT.

The patients will be sent to Operator A for the periodontal examination. Without knowledge of the results of this examination, the patients will be treated by Operators $\mathrm{B}$ and $\mathrm{C}$ for $\mathrm{PT}$, which will involve oral hygiene instructions as well as supragingival and subgingival scaling and planing in up to four sessions. At the end of each session, the patients will be sent to Operator D, who will administer either placebo or active PDT based on the content of the sealed envelope. Thus, both the patients and operator in charge of the periodontal examinations will be blinded to the PDT protocol (a double-blind study).

\section{Examinations for glycemic control and blood count}

Fasting blood sugar and glycated hemoglobin examinations will be performed at baseline as well as 90 and 180 days after the completion of treatment. All examinations will be carried out at the same laboratory affiliated with the university.

\section{Clinical periodontal examination, periodontal treatment and photodynamic therapy}

The clinical periodontal examinations will be performed by a single examiner specialized in periodontics, who will evaluate six regions of each tooth with a $15-\mathrm{mm}$ probe (North Carolina model, Hu-Friedy, Chicago, IL, USA). The following aspects will be recorded: plaque index, bleeding upon probing, probing depth, gingival recession and attachment loss $[4,6]$. Evaluations will be carried out at baseline (prior to initial treatment) as well as 15, 30, 90 and 180 days after the completion of treatment.

PT will consist of oral hygiene instructions, with the monitored orientation of brushing technique and recommendation of the daily use of dental floss. Calculus deposits will also be removed using ultrasound equipment with the appropriate tips for periodontics as well as scaling and planing with dental instruments, as recommended by the AAP [5]. Treatment will be carried out in two to four one-hour sessions with the use of local anesthesia (typically mepivacaine $2 \%$ with 1:100,000 norepinephrine). Gracey periodontal curettes (number 3/4, 5/6, 7/8, 11/12 and 13/14, Hu-Friedy, Chicago, IL, USA) will be used for the removal of the dental calculus. Other biofilm retention factors, such as caries, condemned teeth and poorly fitted restorations, will be removed during the sessions of PT.

PDT will be administered at the end of the PT session at sites with pocket depths equal to or greater than $4 \mathrm{~mm}$. A diode laser (Photon laser III, DMC, São Carlos, Brazil) will be used at the $660 \mathrm{~nm}$ wavelength, with an 


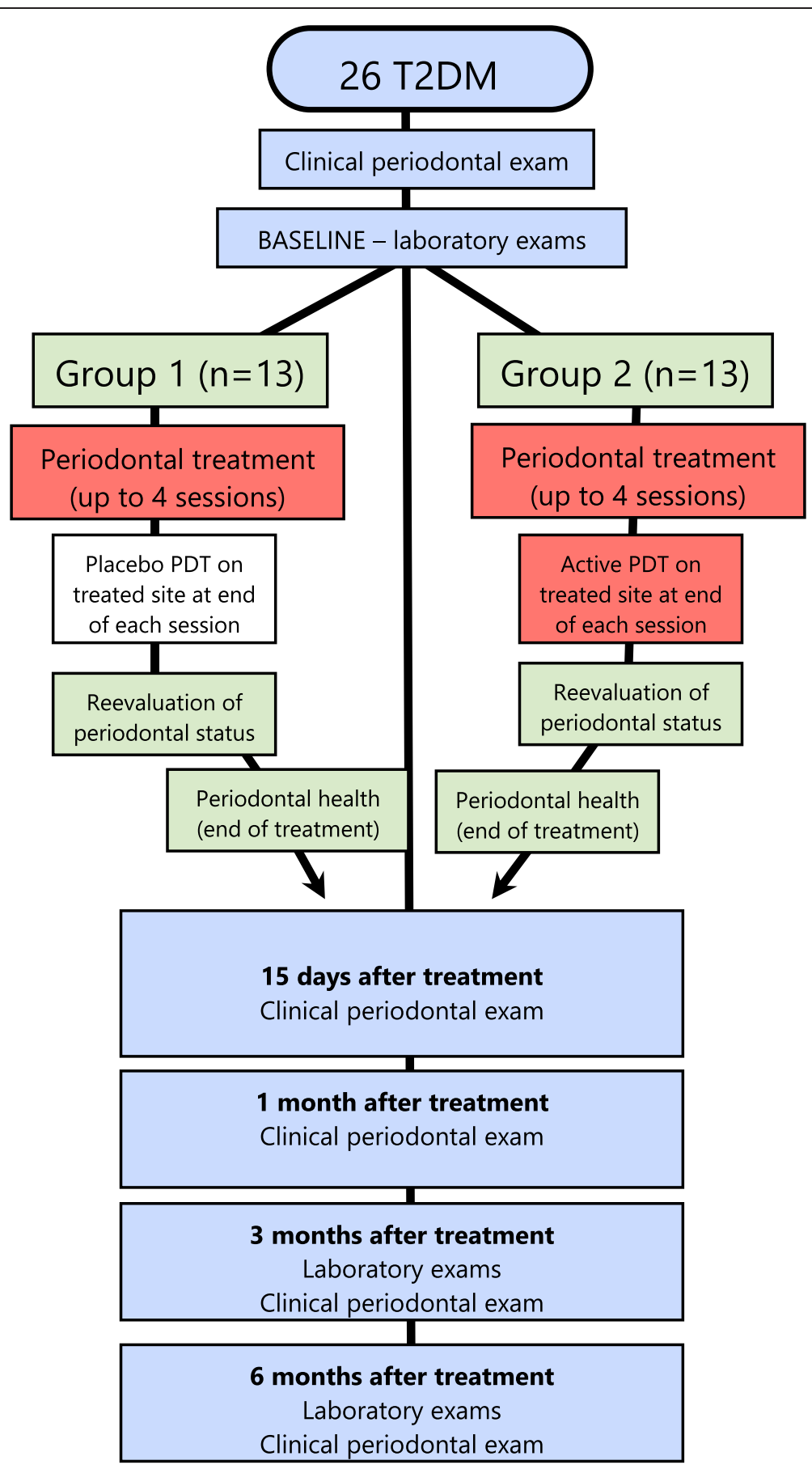

Fig. 1 Flow chart of study design

output power of $100 \mathrm{~mW}$ and 90-s exposure time per point $(9 \mathrm{~J} /$ point $)$. Each irradiation point will have approximately $0.4 \mathrm{~cm}^{2}$, with an energy density per point of $22 \mathrm{~J} / \mathrm{cm}^{2}$ and power density of $250 \mathrm{~mW} / \mathrm{cm}^{2}$. The photosensitizer (Chimiolux $0.005 \%$, DMC, São Carlos, Brazil) will be used, the active ingredient of which is methylene blue at a concentration of $50 \mu \mathrm{g} / \mathrm{mL}$, corresponding to $160 \mu \mathrm{M}$. The photosensitizer will be deposited in each periodontal pocket using a syringe, with application from the fundus of the pocket in the coronal direction. Two minutes will be respected prior to irradiation to allow the medication time to stain all the microbial biofilm. The laser will be applied to the mucosa over the oral epithelium using an optical fiber. Following irradiation, excess medication will be removed with a jet of water. 


\section{Determination of IL-1, IL-6, TNF-alpha, osteocalcin and OPG/RANKL in the crevicular fluid of the periodontal pocket}

Crevicular fluid will be collected from the previously defined sites with a probing depth equal to or greater than $4 \mathrm{~mm}$ that will receive treatment. Collection will be performed with relative isolation using cotton rolls following the removal of the supragingival plaque with periodontal curettes and air drying for five seconds. Absorbent paper strips (PerioPaper, ProFlow Inc., Amityville, NY, USA) will be individually inserted into each site for $30 \mathrm{~s}$ [30]. The collections will be performed by a single researcher. The volume of crevicular fluid samples will be measured using the Periotron 8000 micro-moisture meter (Oraflow Inc., Smithtown, NY, USA). The samples will then be placed in duly identified sterile plastic microtubes, temporarily stored in dry ice and subsequently stored in a freezer at $-80{ }^{\circ} \mathrm{C}$.

\section{Primary and secondary outcome}

The primary outcome variable of the study was clinical attachment level. Secondary outcome variables were probing pocket depth, bleeding on probing, plaque index and level of IL-1, IL-6, tumor necrosis factor (TNF)alpha, osteocalcin and OPG/RANKL.

\section{Data analysis}

The data will be submitted to the appropriate statistical tests for the determination of differences between the groups. Frequency distributions will be determined. The unpaired parametric $t$ test for two samples will be used for data that demonstrate normal distribution, with the level of significance set at $95 \%(P<0.05)$. Data with a log-normal curve will be normalized by the replacement of measures with the base-10 logarithm of their values. In both cases, graphs will be created based on the mean and standard deviation of the data. Data that do not have normal or log-normal distribution will be analyzed using the nonparametric Mann-Whitney test, with the level of significance set to $95 \%(P<0.05)$. These data will be presented in box plots and quartiles constructed based on the median values. All analyses will be performed using the Origin Pro $8^{\mathrm{m} x}$ software program (OriginLab, Northampton, MA, USA).

\section{Trial status}

Patient recruitment is not completed.

\footnotetext{
Abbreviations

AAP: American Academy of Periodontology; ANVISA: The National Health Surveillance Agency of Brazil; DM: diabetes mellitus; IL: interleukin; OPG: osteoprotegerin; PAHO: Pan American Health Organization; PDT: photodynamic therapy; PT: periodontal therapy; RANKL: receptor activator of nuclear factor kappa-B ligand; T2DM: type 2 diabetes mellitus; TNF: tumor necrosis factor; UNINOVE: Nove de Julho University; WHO: World Health Organization.
}

\section{Competing interests}

The authors declare that they have no competing interests.

\section{Authors' contributions}

PV, TOS, and RMG have made substantial contributions to the study design and acquisition of data. AMD, KPSF, RAM-F, and SKB have made substantial contributions to the study design, and data analysis. LHA, VFC, and CPC have made substantial contributions to the study design, acquisition of data, and they have been involved in drafting the manuscript. EE, CMF and RAP have made substantial contributions to the conception and design of the study, acquisition of data, analysis and interpretation of data; they have been involved in drafting the manuscript and revising it critically for important intellectual content; they also have given final approval of the version to be published and they agree to be accountable for all aspects of the work. The authors have read and approved of the final version of the manuscript.

\section{Acknowledgments}

The authors would like to thank Nove de Julho University (UNINOVE).

\section{Author details}

${ }^{1}$ Program in Biophotonics Applied to Health Sciences, University Nove de Julho, UNINOVE, Rua Vergueiro 235/249 - Liberdade, São Paulo, SP 01504-001, Brazil. 'SChool of Medicine, Nove de Julho University UNINOVE, Rua Vergueiro 235/249 - Liberdade, São Paulo, SP 01504-001, Brazil. ${ }^{3}$ School of Dentistry, Nove de Julho University UNINOVE, Rua Vergueiro 235/249 - Liberdade, São Paulo, SP 01504-001, Brazil. ${ }^{4}$ Program in Rehabilitation Science, Nove de Julho University - UNINOVE, Rua Vergueiro 235/249 - Liberdade, São Paulo, SP 01504-001, Brazil. ${ }^{5}$ Medical Sciences, Nove de Julho University UNINOVE, Rua Vergueiro 235/249 - Liberdade, São Paulo, SP 01504-001, Brazil.

Received: 4 November 2014 Accepted: 13 May 2015

Published online: 27 May 2015

\section{References}

1. World Health Organization. Definition and diagnosis of diabetes mellitus and intermediate hyperglycemia. Report of a WHO/IDF Consultation. Geneva, Switzerland: World Health Organization; 2006.

2. Brazil. Diabetes mellitus/ministério da saúde, secretaria de atenção à saúde In: Brazil. Diabetes mellitus/ministério da saúde, secretaria de atenção à saúde. City: Brasilia; 2006. 56.

3. Ramirez JH, Arce RM, Contreras A. Periodontal treatment effects on endothelial function and cardiovascular disease biomarkers in subjects with chronic periodontitis: protocol for a randomized clinical trial. Trials. 2011;12:46.

4. Armitage GC. Periodontal diagnoses and classification of periodontal diseases 2000. Periodontology. 2004;34:9-21.

5. Research, Science and Therapy Committee of the American Academy of Periodontology. Treatment of plaque-induced gingivitis, chronic periodontitis, and other clinical conditions. J Periodontol. 2001;72:1790-800.

6. Armitage GC. Development of a classification system for periodontal diseases and conditions. Ann Periodontol. 1999;4:1-6.

7. D'Aiuto F, Parkar M, Andreou G, Suvan J, Brett PM, Ready D. Periodontitis and systemic inflammation: control of the local infection is associated with a reduction in serum inflammatory markers. J Dent Res. 2004;83:156-60.

8. Sun WL, Chen LL, Zhang SZ, Wu YM, Ren YZ, Qin GM. Inflammatory cytokines, adiponectin, insulin resistance and metabolic control after periodontal intervention in patients with type 2 diabetes and chronic periodontitis. Intern Med. 2011;50:1569-74.

9. Tonetti MS, D'Aiuto F, Nibali L, Donald A, Storry C, Parkar M, et al. Treatment of periodontitis and endothelial function. N Engl J Med. 2007;356:911-20.

10. American Academy of Periodontology-Research, Science and Therapy Committee, American Academy of Pediatric Dentistry. Treatment of plaque-induced gingivitis, chronic periodontitis, and other clinical conditions. Pediatr Dent. 2005;27:202-11.

11. Hamlet SM. Quantitative analysis of periodontal pathogens by ELISA and real-time polymerase chain reaction. Methods Mol Biol. 2010;666:125-40.

12. Kinney JS, Morelli T, Braun T, Ramseier CA, Herr AE, Sugai JV, et al. Saliva/pathogen biomarker signatures and periodontal disease progression. J Dent Res. 2011;90:752-8.

13. Zhang P, Liu J, Xu Q, Harber G, Feng X, Michalek SM, et al. TLR2-dependent modulation of osteoclastogenesis by Porphyromonas gingivalis through 
differential induction of NFATc1 and NF-kappaB. J Biol Chem. 2011;286:24159-69.

14. Christgau M, Palitzsch KD, Schmalz G, Kreiner U, Frenzel S. Healing response to non-surgical periodontal therapy in patients with diabetes mellitus: clinical, microbiological, and immunologic results. J Clin Periodontol. 1998;25:112-24.

15. Lalla E, Kaplan S, Yang J, Roth GA, Papapanou PN, Greenberg S. Effects of periodontal therapy on serum C-reactive protein, sE-selectin, and tumor necrosis factor-alpha secretion by peripheral blood-derived macrophages in diabetes: a pilot study. J Periodontal Res. 2007;42:274-82.

16. Xie Y, Chen J, He J, Miao X, Xu M, Wu X, et al. Antimicrobial resistance and prevalence of resistance genes of obligate anaerobes isolated from periodontal abscesses. J Periodontol. 2014;85:327-34.

17. Tiwari R, Chakraborty S, Dhama K, Wani MY, Kumar A, Kapoor S. Wonder world of phages: potential biocontrol agents safeguarding biosphere and health of animals and humans- current scenario and perspectives. Pakistan J Biol Sci. 2014;17:316-28.

18. Brazil. Monitoramento e prevenção da resistência microbiana em serviços de saúde. In: Brazil. Monitoramento e prevenção da resistência microbiana em serviços de saúde. City: Brasilia; 2006. 22.

19. Rajesh S, Koshi E, Philip K, Mohan A. Antimicrobial photodynamic therapy: an overview. J Indian Soc Periodontol. 2011;15:323-7.

20. Prates RA, Yamada AM, Suzuki LC, Franca CM, Cai S, Mayer MP, et al. Histomorphometric and microbiological assessment of photodynamic therapy as an adjuvant treatment for periodontitis: a short-term evaluation of inflammatory periodontal conditions and bacterial reduction in a rat model. Photomed Laser Surg. 2011;29:835-44.

21. Prates RA, Kato IT, Ribeiro MS, Tegos GP, Hamblin MR. Influence of multidrug efflux systems on methylene blue-mediated photodynamic inactivation of Candida albicans. J Antimicrob Chemother. 2011;66:1525-32.

22. Braham P, Herron C, Street C, Darveau R. Antimicrobial photodynamic therapy may promote periodontal healing through multiple mechanisms. J Periodontol. 2009:80:1790-8.

23. Alvarenga LH, Prates RA, Yoshimura TM, Kato IT, Suzuki LC, Ribeiro MS, et al. Aggregatibacter actinomycetemcomitans biofilm can be inactivated by methylene blue-mediated photodynamic therapy. Photodiagnosis Photodyn Ther. 2015;12:131-5.

24. Konopka K, Goslinski T. Photodynamic therapy in dentistry. J Dent Res. 2007;86:694-707.

25. Chui C, Aoki A, Takeuchi Y, Sasaki Y, Hiratsuka K, Abiko Y, et al. Antimicrobial effect of photodynamic therapy using high-power blue light-emitting diode and red-dye agent on Porphyromonas gingivalis. J Periodontal Res. 2013;48:696-705

26. Fimple JL, Fontana CR, Foschi F, Ruggiero K, Song X, Pagonis TC, et al. Photodynamic treatment of endodontic polymicrobial infection in vitro. J Endod. 2008;34:728-34.

27. Campos GN, Pimentel SP, Ribeiro FV, Casarin RC, Cirano FR, Saraceni $\mathrm{CH}$ et al. The adjunctive effect of photodynamic therapy for residual pockets in single-rooted teeth: a randomized controlled clinical trial. Lasers Med Sci. 2013;28:317-24.

28. Braun A, Dehn C, Krause F, Jepsen S. Short-term clinical effects of adjunctive antimicrobial photodynamic therapy in periodontal treatment: a randomized clinical trial. J Clin Periodontol. 2008;35:877-84.

29. Correa FO, Goncalves D, Figueredo CM, Bastos AS, Gustafsson A, Orrico SR. Effect of periodontal treatment on metabolic control, systemic inflammation and cytokines in patients with type 2 diabetes. J Clin Periodontol. 2010;37:53-8.

30. Loe $H$, Holm-Pedersen P. Absence and presence of fluid from normal and inflamed Gingivae. Periodontics. 1965;3:171-7.

\section{Submit your next manuscript to BioMed Central and take full advantage of:}

- Convenient online submission

- Thorough peer review

- No space constraints or color figure charges

- Immediate publication on acceptance

- Inclusion in PubMed, CAS, Scopus and Google Scholar

- Research which is freely available for redistribution 